\title{
A New Assessment of Activities of Daily Living for Thai Stroke Patients
}

\author{
Sopida Apichai*, Pisak Chinchai, Jananya P. Dhippayom, \\ and Peeraya Munkhetvit
}

\begin{abstract}
Department of Occupational Therapy, Faculty of Associated Medical Sciences, Chiang Mai University, Chiang Mai 50200, Thailand

*Corresponding author. E-mail: sopida.apichai@cmu.ac.th https://doi.org/10.12982/CMUJNS.2020.0012
\end{abstract}

Received: January 27, 2019

Revised: May 10, 2019

Accepted: June 17, 2019

\begin{abstract}
This study aimed to develop the Activities of Daily Living Assessment Tool (ADL-AT) for Thai stroke patients and to examine its psychometric properties. 45 stroke patients and 45 normal subjects were selected by purposive sampling. Raters were occupational therapists experienced with stroke patients and received ADL-AT for Thai stroke patients training. To measure inter-rater reliability, stroke patients were assessed by two raters at the same time. To assess the test-retest reliability, stroke patients were retested at a time interval within 5 to 7 days. Intraclass correlation coefficients (ICC) model 3, $k$ and 3, 1 were used to determine inter-rater and test-retest reliability, respectively. Internal consistency was evaluated using Cronbach's alpha which was conducted to study construct validity. The independent samples t-test was used to measure the test's ability to discriminate between stroke patients and normal subjects. The present study found that ADL-AT for Thai stroke patients had shown an excellent inter-rater and test-retest reliability $($ ICC $=.98, .93$, respectively). The analysis of Cronbach's alpha coefficient revealed high internal consistency $(\alpha=.88)$. In addition, this tool displayed a positive correlation with Barthel index $(r=0.9 ; P<0.001)$. In known-groups validity, stroke patients obtained lower ADL-AT for Thai stroke patients scores compared to normal subjects $(P<0.001$.). The results of this study suggest that the ADL-AT for Thai stroke patients is known to be reliable and valid and recommended to further the services system.
\end{abstract}

Keywords: Stroke, Activities of daily living, Reliability, Validity 


\section{INTRODUCTION}

A stroke is one of the most common causes leading to sensory-motor deficits and disability in activities of daily living (Hsueh et al., 2012). In Thailand, there are more than 250,000 new cases of stroke each year and the average age of these patients is 65 years (Suwanwela, 2014). Thai stroke patients are usually admitted to hospital for a short-period of time ranging between three days and two weeks. After that, a large number of patients are discharged from hospital and return to their home and are taken care of by their caregivers which are family members or relatives (Suwanwela, 2014). Numerous investigations have exhibited functional disabilities that are chance variables of hospitalization, increased healthcare expenditures, institutional residence, and poor quality of life (Spillman, 2004; Cesari et al., 2008; Li et al., 2010). Therefore, enabling activities of daily living independence during rehabilitation can lead to a better quality of life for stroke patients (Fawcus, 2008).

In past decades, various activities of daily living assessment tools have been developed for stroke patients including the Functional Independence Measure (FIM) (Granger et al., 1986), the Stroke Impairment Assessment Set (SIAS) (Chino et al., 1994), Barthel Index (BI) (Mahoney and Barthel, 1965), and the modified BI (MBI) (Collin et al., 1988; Shah et al., 1989) etc. Each one of these assessment tools has various advantages and disadvantages. For instance, a few of them are sensitive to changes, therefore require advanced knowledge for their administration. A few of the assessment tools used are easier than the others; however, not as detailed as the others (Ohura et al., 2017). Moreover, activities of daily living in specifically instrumental activities of daily living measures are culture-sensitive (Fillenbaum, 1984). Indeed, even inside a country there might be culturally decided contrasts, most remarkably reflecting gender roles (Heikkinen et al., 1983). Regarding the cultural contrasts that may be expected, significant contrasts between developing and developed countries in foundation and resources require even general tasks, such as cleaning, cooking, personal care and community mobility, to be performed in various ways. As a consequence, activities of daily living assessment developed in one setting may not measure apparently common activities in the same way as another setting (Fillenbaum et al., 1999). Importantly, a culturally appropriate approach plays an importance role to help stroke patients to recover their functional ability by enabling them to perform purposeful and meaningful task. Choosing relevant and meaningful activities which encounter the needs of the patient lead to the enhancement of a satisfying and good quality of life for stroke patients.

Occupational therapy is a client-centered health profession concerned with promoting health and well-being through occupation (World Federation of Occupational Therapists, 2013). In Thailand, there are three common activities of daily living assessment tools used by occupational therapists such as (1) Sirindhron National Medical Rehabilitation Center-Functional Assessment 
(SMNRC-Functional Assessment) (Suwapan et al., 2005), (2) Barthel index (BI) (Wolf and Rognstad, 2013), and (3) modified BI (MBI) (Loharjun et al., 2010). However, there are no tools which consider the activities that the patients could perform to increase participation in roles such as care of others, child rearing, and community mobility. Therefore, the purpose of this study is to develop an activities of daily living assessment tool for Thai stroke patients that is appropriate for Thai culture and to evaluate its validity and reliability.

\section{MATERIALS AND METHODS}

This study was divided into two phases: (1) development of the activities of daily living assessment tool (ADL-AT) for Thai stroke patients and (2) examination of psychometric properties in terms of; inter-rater reliability, testretest reliability, construct validity, and concurrent validity. This study was reviewed and approved by the human ethics committees of the Faculty of Associated Medical Sciences, Chiang Mai University (approval number: AMSEC-59EX-052) and the Chiang Mai Neurological Hospital (approval number: EC 003-61). All participants signed a consent form prior to the participation.

\section{Participants}

The participants were recruited from two hospitals and three community rehabilitation centers in Chiang Mai province, comprising: Chiang Mai Neurological Hospital, Sarapeeborvonpattana Hospital, Nongpakrung Community Rehabilitation Center, Huaykiang-Temple Community Rehabilitation Center, and Nong-Kwai Community Rehabilitation Center. Inclusion criteria for participants were (1) diagnosis of stroke (based on physician's diagnosis), (2) having stroke $\geq 24$ hours (subacute stroke), (3) 18 years of age or older, and (4) with or without communication and cognitive impairment (5) able to follow verbal instructions to complete the assessment. Patients with the following conditions were excluded (1) major comorbidities that might result in incomplete assessment such as depression (obtaining a score less than 7 on 9-Depression Test Questionnaire: 9Q) or hallucination (2) unstable medical conditions that might result in incomplete assessment such as acquiring an injury, or severe cardiac or pulmonary disorder, or (3) did not wish to continue participating in this study.

In the first phase, five patients were purposely selected as the same criteria as described above for trial using the assessment tool. In the second phase, for investigating the psychometric properties, the estimated sample size was calculated using $G^{*}$ power (power of test $=0.95$, level of significance $\alpha=.05$, effect size $=0.5$ ). Therefore, 45 stroke patients who met the inclusion criteria and 45 normal subjects who matched with the stroke patients were included for this study. 


\section{Procedure}

The procedure of this study was divided into two phases. The first phase was the development of ADL-AT for Thai stroke patients and the second phase was testing of the psychometric properties of the newly developed instrument.

Phase 1: Developing the ADL-AT for Thai stroke patients. The purpose of this phase was to construct items covering the board spectrum of activities of daily living function regarding Thai culture, and create a scoring system. We developed a record form of activities of daily living assessment tool by extracting items from literature review. However, because this tool was developed for occupational therapists who worked with Thai stroke patients, the administration of activities of daily living items was conducted based on occupational therapy practice framework (OTPF): domain \& process and Thai culture. After several rounds of panel discussion with our team (three occupational therapy academics who were experienced with stroke patients for more than 20 years), the final number and content of items were determined. These discussions resulted in the development of two main sets of activities including: (1) activities that were oriented toward taking care of one's own body known as basic activities of daily living (BADL), and (2) activities that were oriented toward interacting with the environment and that are often complex-generally optional in nature known as instrumental activities of daily living (IADL). The BADL items were subdivided into three main sub sets: (1) self-care (9 items), (2) functional mobility (15 items), and (3) sexual expression (1 item). Fifteen items of functional mobility were subdivided as bed mobility (4 items), transferring (9 items), and locomotion (2 items). The IADL were divided into 12 items. Therefore, the first draft of ADLAT for Thai stroke patients, a total of 37 items 25 BADL items and 12 IADL items), was developed at this stage.

The scoring system was undertaken after generating the items. The scale ranges from 1 to 5 , where ' 1 ' means 'dependence' and ' 5 ' means 'independence'. The total scores for all 37 items and for each of the two parts separately, which are BADL and IADL, were calculated as a percentage. The scoring method is as follows:

1. The therapist, grades 1 to 5 according to the level of ability of the patient in the topics that are evaluated

2. The therapist does not need to evaluate all the topics included in the assessment form. If any activity is not required by the patient, it is not applicable (N/A).

3. Divide each individual score by full score. The recipients should be able to do all the activities on the topics that are rated at level 5. For example, if evaluating 30 topics, the full score would be 150 .

4. Calculate the score to the percentage.

The total score may range from $0-100 \%$. A lower score indicates a higher level of dependence. 
In this study, the first draft of activities of ADL-AT for Thai stroke patients was examined for content validity by three expert occupational therapists who have worked with stroke patients for more than 5 years. The average index of item-objective congruence (IOC) was 0.75 which indicates good content validity (Turner and Carlson, 2003). However, the IOC index for each item ranged between $0.33-1.00$. The items with IOC index that were more than or equal to 0.5 were accepted and items with IOC index less than 0.5 were removed or revised according to the comments of the experts and a second draft was subsequently developed. In the present study, items "transfer from wheelchair $(\mathrm{w} / \mathrm{c})$ to toilet and from toilet back to $\mathrm{w} / \mathrm{c}$ " have been removed because it is a part of item "bathing, showering". Moreover, item "transfer from w/c to tub/shower and tub/shower to w/c" was also removed because it is not appropriate with Thai culture where majority of people do not use bathtub at home. To assess the problems that might be found when using this tool, the second draft was trial assessment in 5 stroke patients (who were purposely selected from two hospitals and three community rehabilitation centers) before developing a final draft. Therefore, the final draft of the 35-items activities of daily living assessment was considered valid (Table 1).

Phase 2: Testing of psychometric properties. The aim of this phase was to evaluate the validity and reliability of the ADL-AT for Thai stroke patients. This phase was conducted immediately after the development of the assessment tool in phase 1. Thus, rater B (research assistants who had 5 years of experience working with stroke patients) received 4 hours of training from the rater $\mathrm{A}$ (principle investigator, an experienced ADL assessment tool user). The contents of rater training are as follows: First, they spend one and a half hours introducing the development of the activities of daily living assessment tool for Thai stroke patients to rater B and allow rater B to familiarize themselves with the items. Second, one hour is allotted for raters to practice with administration on at least 10 case studies. Third, they spend one and a half hours on discussing the scoring results with the principle investigator (the master rater). After the training session, both raters individually evaluated 5 patients while the principle investigator observes and scores at the same time. Then the rater' assessment procedures and scoring results are checked by the principle investigator to ensure that the procedures and results are satisfactory. 
Table 1. The items of the activities of daily living assessment for Thai stroke patients.

\begin{tabular}{ll}
\hline BADL (23-items) & IADL (12-items) \\
\hline 1. Bathing, showering & 1. Care of others \\
2. Toileting and toilet hygiene & 2. Child rearing \\
3. Bowel and bladder control & 3. Care of pets \\
4. Dressing-Upper part & 4. Communication management \\
5. Dressing-Lower part & 5. Community mobility \\
6. Swallowing/eating & 6. Financial management \\
7. Feeding & 7. Health management and maintenance \\
8. Personal device care & 8. Home establishment and management \\
9. Personal hygiene and grooming & 9. Meal preparation and cleanup \\
10. In-bed mobility & 10. Religious and spiritual activities and \\
11. Rolling & expression \\
12. Sitting up & 11. Safety and emergency maintenance \\
13. Standing up & 12. Shopping \\
14. Floor to bed, bed to floor & \\
15. Floor to chair, chair to floor & \\
16. Floor to toilet, toilet to floor & \\
17. Floor to w/c, w/c to floor & \\
18. w/c to bed, bed to w/c & \\
19. w/c to chair, chair to w/c & \\
20. w/c to car, car to w/c & \\
21. Wheelchair locomotion & \\
22. Walking locomotion & \\
23. Sexual expression &
\end{tabular}

Reliability. For inter-rater reliability, 45 participants, who met the inclusion criteria, were assessed with activities of daily living assessment by two raters at the same time in one session. To prevent the raters scoring being affected by each other's, raters A and B independently scored the participants and did not communicate with each other or the principle investigator throughout the study. For test-retest reliability, 5 to 7 days interval between the $1^{\text {st }}$ and $2^{\text {nd }}$ assessment was given to prevent recall of raters regarding participant's level of activities of daily living. Moreover, during the $2^{\text {nd }}$ assessment session, raters were blinded to the results of the $1^{\text {st }}$ session.

Validity. To determine the concurrent validity of activities of ADL-AT for Thai stroke patients, Barthel index was assessed for all participants in the first assessment by one rater ( 2 days later from the $1^{\text {st }}$ session). To investigate the construct validity, 45 normal subjects were assessed by ADL-AT for Thai stroke patients. 


\section{Statistical analysis}

Descriptive statistics were used to summarize the information on the participants (sociodemographic data). Inter-rater and test-retest reliability for total items of ADL-AT for Thai stroke patients were calculated by Intraclass correlation coefficients $\left(\mathrm{ICC}_{3, \mathrm{k}}\right.$ and $\mathrm{ICC}_{3,1}$, respectively). ICC value $\geq 0.8$ and between 0.6-0.8 indicates excellent and moderate reliability, respectively (Hsueh et al., 2001). Internal consistency was assessed using Cronbach's $\alpha$ coefficient. The minimum acceptable value for $\alpha$ was 0.7 (Hsueh et al., 2001).

To investigate the concurrent validity of the ADL-AT for Thai stroke patients, the Pearson Correlation coefficient (95\% limits of agreement) was used to examine the strength of association among the total ADL scores, BADL and IADL scores of the ADL-AT for Thai stroke patients and those of the Barthel index. The Barthel index was treated as a gold standard measure in this study. The criteria for evaluating the correlation coefficient was as follows: Pearson's $r$ coefficient of .80 or above is considered to be excellent, .60-.79 moderate, and less than .60 is considered poor (Portney and Watkins, 2000). The known-group construct validity of activities of daily living assessment tool for Thai stroke patients was evaluated by performing the independent samples $t$-test to examine the difference of the ADL-AT for Thai stroke patients scores between the people with and without a stroke. A significant difference for analysis was set at $P<.05$. Statistical analysis was performed with SPSS Version 17.

\section{RESULTS}

Table 2. General characteristics of participants in the study.

\begin{tabular}{lccc}
\hline Characteristics & Stroke $(\mathbf{n}=\mathbf{4 5})$ & Control $(\mathbf{n}=\mathbf{4 5})$ & $\boldsymbol{P}$ value \\
\hline Gender & & & \\
$\quad$ Male & $31(68.89)$ & $31(68.89)$ & 1 \\
$\quad$ Female & $14(31.11)$ & $14(31.11)$ & \\
Age (years) & $60.60 \pm 9.87$ & $60.09 \pm 7.07$ & 0.778 \\
$\quad($ minimum- maximum) & $(60-85)$ & $(65-83)$ & \\
Type of stroke & & & \\
$\quad$ Ischemic & $36(80)$ & & \\
$\quad$ Hemorrhagic & $9(20)$ & & \\
Time after the onset (months) & $3.47 \pm 4.21$ & & \\
Note: $\begin{array}{l}\text { Data are expressed as } n(\%), \text { expected average age, and elapsed time since the onset, which are expressed } \\
\text { as the mean } \pm \text { SD and/or minimum- maximum. }\end{array}$ &
\end{tabular}

Forty-five stroke patients and 45 healthy control subjects were included in this study. Their general characteristics are shown in Table 2. The participants were well matched in terms of age and gender. Of those, 31 stroke patients were male $(68.89 \%)$. The mean age of all patients was $60.60(\mathrm{SD}=9.87)$ years. 
Regarding stroke types, 36 patients had ischemic stroke, while 9 patients had hemorrhagic stroke. The median time after stroke was 3.47 years.

On the other hand, the mean age control subjects was $60.09(\mathrm{SD}=7.07)$ years, and $68.89 \%$ of the control subjects were male. No significant difference was observed between the two groups in terms of their baseline general characteristics.

Table 3. Functional disability in activities of daily living among stroke patients.

\begin{tabular}{lcc}
\hline Items & $\begin{array}{c}\text { Functional independence } \\
\mathbf{n}(\boldsymbol{\%})\end{array}$ & $\begin{array}{c}\text { Functional disability } \\
\mathbf{n}(\mathbf{\%})\end{array}$ \\
\hline BADL & $40(88.9)$ & $5(11.1)$ \\
Feeding & $27(60.0)$ & $18(40.0)$ \\
Bathing & $26(57.8)$ & $19(42.2)$ \\
Grooming & $28(62.2)$ & $17(37.8)$ \\
Dressing & $37(82.2)$ & $8(17.8)$ \\
Bowels & $37(82.2)$ & $8(17.8)$ \\
Bladder & $29(64.4)$ & $16(35.6)$ \\
Toilet use & $27(60.0)$ & $18(40.0)$ \\
Mobility (on level surfaces) & $26(57.8)$ & $19(42.2)$ \\
Stairs & & \\
IADL & $28(62.2)$ & $17(37.8)$ \\
Ability to use telephone & $24(53.3)$ & $21(46.7)$ \\
Shopping & $9(20.0)$ & $36(80.0)$ \\
Food preparation & $19(42.2)$ & $26(57.8)$ \\
Housekeeping & $31(68.9)$ & $14(31.1)$ \\
Ability to handle finances & $26(57.8)$ & $19(42.2)$ \\
Responsibility for own & $20(44.4)$ & $25(55.56)$ \\
medication & & \\
Mode of transportation & & \\
\hline Note: Date are expressed as n (\%). BADL: basic activities of daily living; IADL: instrumental activities of daily \\
living. & &
\end{tabular}

Table 3 shows the proportion of functional disability in each ADL item among stroke patients. Each item was evaluated either as functionally independent or functionally disabled requiring assistance. In stroke patients, there is greater prevalence in disability in food preparation, housekeeping, and transport. 


\section{Reliability}

Inter-rater reliability for ADL total scores, BADL and IADL scores were $.98, .95$, and .98 , respectively, indicating excellent reliability. Moreover, test-retest reliability also showed excellent reliability $\left(\mathrm{ICC}_{3,1}\right.$ for ADL total score, BADL and IADL scores were .93, .95, and .83, respectively) (Table 4).

The internal consistency coefficient obtained were high, with Cronbach's alpha $=0.88$ for the total 35-items of ADL, 0.72 for the 23-items of BADL, and 0.86 for the 12-items of IADL, suggesting that these items are sufficiently homogeneous (Table 4).

Table 4. Inter-rater, test-retest reliability, and internal consistency of activities of daily living assessment tool for Thai stroke patients.

\begin{tabular}{lccc}
\hline & Inter-rater & Test-retest & $\begin{array}{c}\text { Cronbach's Alpha } \\
\text { if item deleted }\end{array}$ \\
\cline { 2 - 3 } BADL & ICC $\mathbf{3 , \mathbf { k }} \mathbf{( 9 5 \% \mathbf { C I } )}$ & $\left.\mathbf{I C C}_{\mathbf{3}, \mathbf{1}} \mathbf{( 9 5 \%} \mathbf{C I}\right)$ & .75 \\
\hline IADL & $.98(.96-.99)$ & $.95(.92-.98)$ & .86 \\
\hline Total ADL & $.95(.91-.97)$ & $.83(.71-.90)$ & .88 \\
\hline
\end{tabular}

Note: BADL: basic activities of daily living; IADL: instrumental activities of daily living; ADL: activities of daily living; ICC: intraclass correlation coefficients; 95\% CI: 95\% confidence interval.

\section{Validity}

As shown in Table 5, concurrent validity was calculated by evaluating the correlation between the ADL-AT for Thai stroke patients and Barthel index. An excellent correlation was found between the total score of Barthel index with BADL and the total ADL scores of ADL assessment for Thai stroke patients $(r=0.94$ and 0.90 respectively, $P<0.001)$. Also, there was moderate correlation $(r=0.60)$ between the total score of Barthel index and the IADL score of ADLAT for Thai stroke patients.

One method of determining the construct validity is to investigate the known group differences. Here, our hypothesis was that stroke patients, compared to normal subjects who do not have such problems, have less ability for functional independence. Table 5, using independent samples $t$-test, demonstrated that all ADL assessment scores discriminated between normal subjects and stroke patients. These results indicated that the ADL assessment is targeted to stroke patients. 
Table 5. Discriminant concurrent and construct validity of activities of daily living assessment tool for Thai stroke patients.

\begin{tabular}{lccc}
\hline \multicolumn{1}{c}{ Characteristics } & $\begin{array}{c}\text { Barthel index } \\
\text { score; } r\end{array}$ & $\begin{array}{c}\text { Stroke (n=45) } \\
\text { mean (SD) }\end{array}$ & $\begin{array}{c}\text { Control (n=45) } \\
\text { mean (SD) }\end{array}$ \\
\hline ADL assessment score & & & \\
BADL & $0.94^{*}$ & $87.40(17.37)$ & $100.00(0.00)^{\dagger}$ \\
IADL & $0.60^{*}$ & $81.65(24.21)$ & $99.63(0.01)^{\dagger}$ \\
Total ADL & $0.90^{*}$ & $85.24(18.15)$ & $99.86(0.39)^{\dagger}$
\end{tabular}

Barthel index score (mean $\pm \mathrm{SD})$ : $\quad 76.18 \pm 32.02$

Note: ADL: activities of daily living; BADL: basic activities of daily living; IADL: instrumental activities of daily living; Data are expressed as mean (SD). ${ }^{*} P<0.001$ for strength of association between the scores of ADT-AT for Thai stroke patients and those of the Barthel index (Pearson Correlation coefficient). ${ }^{\dagger} P<$ 0.001 for the difference between stroke and control groups for ADL total scores, BADL and IADL scores from ADL-AT for Thai stroke patients (independent samples $t$-test).

\section{DISCUSSION}

Currently, there is no comprehensive standard tool for occupational therapists to evaluate activities of daily living functioning among Thai stroke patients. The activities of daily living assessment tool (ADL-AT) for Thai stroke patients was originally based on the occupational therapy practice framework (OTPF) and Thai culture. Items covered both BADL and IADL. Therefore, ADLAT for Thai stroke patients provides a means of identifying functional ability to perform meaning and purpose and purposeful activities in Thai context. The administration times of this tool range from 10-15 minutes which includes data gathering through direct observation or interviewing patients or care givers.

The results of this study exhibited that the ICC value (total $\mathrm{ADL}=.98$, $\mathrm{BADL}=.98, \mathrm{IADL}=.95$; Table 4 ) obtained from the inter-rater assessments was excellent, indicating that ADL-AT for Thai stroke patients has a near perfect inter-rater agreement. These findings are due to three reasons. First, the ADL-AT for Thai stroke patients has a good content validity. In the present study, an initial set of items was developed after literature review, and discussion with professional experts whose field work is familiar with the stroke patient and Thai culture. These procedures brought about the development of a lengthy set of activities, including well understood items, easy scoring, and necessary seasonal tasks, in which most stroke patients in the Thai community might be expected to engage in. Second, the raters have sufficient training before examination. Third, the experience of the raters may have an influence with the inter-rater reliability. In the present study, both of the raters were occupational therapists who have worked with stroke patients for more than 5 years. Therefore, both of them have good judgment and scoring the functional ability of the patients. Consequently, a strong agreement in the scoring between both raters occurred. However, the 
agreement rate of IADL was lower than BADL in this study. This is because of the distinction in the assessment techniques: almost all IADL items were evaluated by self-reporting from the subjects rather than direct observation of their performance by therapists. These results suggested that the ADL-AT for Thai stroke patients produces consistent results for representing patients' activities of daily living functions, regardless of whether the ADL-AT for Thai stroke patients is administered by an individual rater or by various raters.

Results of the test-retest study showed higher stability of the measure over time (Table 4), indicating that ADL-AT for Thai stroke patients has excellent reliability over a 5-7 day interval. These time intervals were appropriate because it was believed that the intervals were too short for clinical change in patients who were believed to be in a steady state. The average illness duration of the patients in this study was 3.47 years, who were chronic stroke patients who had showed no natural recovery (Park, 2018). Moreover, with at least 5 days between assessment completion, sufficient time would have elapsed to minimize the bias associated with the recollection of a previous response. A lower level of ICC value of IADL (.83) than BADL (.95) were found in this study. This lower level of agreement can be explained by the possibility of the data collection effect by the subject. It is well known that, IADL is influenced by environmental, educational, societal, and cultural factors, which is more complicated than BADL (Walter-Ginzburg et al., 2001). In the present study, most of the IADL information was obtained from patients or the care givers interview (subjective data). Unlike direct observation (objective data), the differences in a patient's answer have the potential to alter their performance between sessions (Choi et al., 2014).

The ADL-AT for Thai stroke patients has adequate internal consistency as demonstrated by the high value of the alpha coefficient (Table 4). This means that all items of an instrument measure the same characteristics indicating item homogeneity. Due to a well-established framework and expert panel during instrumental development, a new instrument that assesses a single construct was developed. In this study, concurrent validity with the Barthel index showed a highly significant positive correlation. Although results of the correlation between each score and total Barthel index score indicated a statistically significant association, only the IADL score had a relatively moderate correlation with the total score of Barthel index (0.60; Table 5). A possible reason for this result may be associated with gold standard choices. Even though the Barthel index has satisfactory psychometric properties in stroke patients (Oveisgharan et al., 2006). Barthel index evaluates functional ability in 10 items including bathing, grooming, bowels, bladder, toilet use, dressing, feeding (as self-care items); and transfer (bed to chair and back), mobility, stair up and down. All of these items were categorized as BADL area leading to a finding that a moderate correlation was found when compared between the total score of Barthel index and IADL score of ADL-AT for Thai stroke patients. There are a few strategies 
to confirm the construct validity. One of them is the known-groups technique. In this approach, different groups of individuals fill in the research instrument and then the groups' results are compared. As expected, ADL-AT for Thai stroke patients scores (total, BADL, and IADL) were significantly lower in stroke patients compared to healthy controls (Table 5). It could indicate that the ADLAT for Thai stroke patients could differentiate the groups with different ability to perform daily functions.

It is well recognized that a clinically useful activities of daily living measure must be scientifically sound in terms of two basic psychometric properties: reliability and validity. Therefore, all validity and reliability testing in the psychometric evaluation confirmed that the ADL-AT for Thai stroke patients was a newly developed assessment tool that was quantifiable to measure functional ability of Thai stroke patients. The ADL-AT for Thai stroke patients has three advantages. First, the instrument included a variety of activities that cover the number of activities that a patient needs to do to occupy role and need. Second, it requires only the least amount of time in collecting data and interpreting results. Although the ADL-AT for Thai stroke patients evaluated functional ability of the patients using multiple activities, this instrument investigated only items that patients have satisfaction with the patient's role. For example, when the activity is unnecessary, the patients can be scored as not applicable (N/A) to reduce assessment burden. Moreover, it not necessary to sum the score; we can divide the domain score independently. Third, ADL-AT for Thai stroke patients helps occupational therapists the same as other health professionals to assess activities of daily living in stroke patients in comprehensive way, and having a pronounced decreasing ceiling effect. It is not only applied for evaluating BADL function but also it evaluates very mild complexity functions impairment or nearly recovered stroke.

A number of limitations was found in the present study. Generalization of the results of this study may be limited by the convenient sampling of participants. The majority of the participants were outpatients living in the community, and most of the stroke patients were at the chronic stage. The BADL items are more appropriate for inpatients when compared with IADL items. Consequently, the generalization of the results in the present study may be limited. Given the correlation of the ADL-AT for Thai stroke patients and Barthel index, as observed in this study, further studies to investigate the correlation of ADL-AT for Thai stroke patients and with another standard IADL assessment tool, i.e., Lawton Instrumental Activities of Daily Living Scale, Katz index etc., is warranted (Khoei et al., 2013). Moreover, the number of stroke patients was not considered for evaluated factor analysis in the present study which is recommended to be addressed in future studies. The ADL-AT for Thai stroke patients was confirmed to be a useful evaluation instrument in that its inter-rater and test-retest agreement rate, internal consistency, and validity were high. Therefore, the ADL-AT for 
Thai stroke is a reliable and valid clinical instrument for assessing the activities of daily living among Thai stroke patients.

\section{CONFLICTS OF INTEREST}

The authors declare that there is no conflict of interest.

\section{ACKNOWLEDGEMENTS}

This study was granted by the Faculty of Associated Medical Sciences. Chiang Mai University, Chiang Mai, Thailand.

\section{REFERENCES}

Cesari, M., Onder, G., Zamboni, V., Manini, T., Shorr, R.I., Russo, A., Bernabei, R., Pahor, M., and Landi, F. 2008. Physical function and selfrated health status as predictors of mortality: results from longitudinal analysis in the ilSIRENTE study. BMC Geriatr. 8: 34. https://doi.org/ 10.1186/1471-2318-8-34

Chino, N., Sonoda, S., Domen, K., Saitoh, E., and Kimura, A. 1994. Stroke Impairment Assessment Set (SIAS): a new evaluation instrument for stroke patients. The Japanese Journal of Rehabilitation Medicine. 31(2): 119-125. https://doi.org/10.2490/jjrm1963.31.119

Choi, Y.M., Dobson, F., Martin, J., Bennell, K.L., and Hinman, R.S. 2014. Interrater and intrarater reliability of common clinical standing balance tests for people with hip osteoarthritis. Physical Therapy. 94(5): 696-704. https://doi.org/10.2522/ptj.20130266

Collin, C., Wade, D.T., Davies, S., and Horne, V. 1988. The Barthel ADL Index: a reliability study. International Disability Studies. 10(2): 61-63.https:// doi.org/10.3109/09638288809164103

Fawcus, R. 2008. Stroke rehabilitation: a collaborative approach. UK: Blackwell Science Ltd.

Fillenbaum, G., Chandra, V., Ganguli, M., Pandav, R., Gilby, J.E., Seaberg, E.C., Bell, S., Baker, C., Echement, D.M., and LM, N. 1999. Development of an activities of daily living scale to screen for dementia in an illiterate rural older population in India. Age Ageing. 28(2): 161-168. https://doi.org/ 10.1093/ageing/28.2.161

Fillenbaum, G.G. 1984. The wellbeing of the elderly. Approaches to multidimensional assessment. WHO Offset Publication. 84: 1-99.

Granger, C.V., Hamilton, B.B., Keith, R.A., Zielezny, M., and Sherwin, F.S. 1986. Advances in functional assessment for medical rehabilitation. 1(3): 59-74. https://doi.org/10.1097/00013614-198604000-00007 
Heikkinen, E., Waters, W., and Brzezinski, Z. 1983. The elderly in Eleven Countries. Geneva: WHO Regional Office for Europe.

Hsueh, I.P., Lee, M.M., and Hsieh, C.L. 2001. Psychometric characteristics of the Barthel activities of daily living index in stroke patients. Journal of the Formosan Medical Association. 100(8): 526-532.

Hsueh, I.P., Wang, C.H., Liou, T.H., Lin, C.H., and Hsieh, C.L. 2012. Test-retest reliability and validity of the comprehensive activities of daily living measure in patients with stroke. Journal of Rehabilitation Medicine. 44(8): 637-641. https://doi.org/10.2340/16501977-1004

Khoei, M.A., Akbari, M.E., Sharifi, F., Fakhrzadeh, H., and Larijani, B. 2013. Translation and validation of the activities of daily living scale with Iranian elderly cancer patients treated in an oncology unit. Asian Pacific journal of cancer prevention. 14(5): 2731-2737. https://doi.org/10.7314/APJCP. 2013.14.5.2731

Li, L., Chang, H.J., Yeh, H.I., Hou, C.J.Y., Tsai, C.H., and Tsai, J.P. 2010. Factors associated with leisure participation among the elderly living in long-term care facilities. International Journal of Gerontology. 4(2): 69-74. https:// doi.org/10.1016/S1873-9598(10)70026-0

Loharjun, B., Wannapira, P., Palivanit, J., and Cumjun, K. 2010. Reliability of Modified Barthel Index (Thai Version) Assessment in Stroke Patients. 25.

Mahoney, F.I., and Barthel, D.W. 1965. Functional evaluation: the Barthel index. Maryland State Medical Journal. 14: 61-65.

Ohura, T., Hase, K., Nakajima, Y., and Nakayama, T. 2017. Validity and reliability of a performance evaluation tool based on the modified Barthel index for stroke patients. BMC Medical Research Methodology. 17(1): 131. https://doi.org/10.1186/s12874-017-0409-2

Oveisgharan, S., Shirani, S., Ghorbani, A., Soltanzade, A., Baghaei, A., Hosseini, S., and Sarrafzadegan, N. 2006. Barthel index in a Middle-East country: translation, validity and reliability. Cerebrovascular Diseases. 22(5-6): 350-354. https://doi.org/10.1159/000094850

Park, C. S. 2018. The test-retest reliability and minimal detectable change of the short-form Barthel index (5 items) and its associations with chronic strokespecific impairments. Journal of Physical Therapy Science. 30(6): 835839. https://doi.org/10.1589/jpts.30.835

Portney, L., and Watkins, M. 2000. Foundation of clinical research: application to practice. New Jersey: Prentice Hall Health.

Shah, S., Vanclay, F., \& Cooper, B. 1989. Improving the sensitivity of the Barthel Index for stroke rehabilitation. Journal of Clinical Epidemiology. 42(8): 703-709. https://doi.org/ 10.1016/0895-4356(89)90065-6

Spillman, B.C. 2004. Changes in elderly disability rates and the implications for health care utilization and cost. Milbank Quarterly. 82(1): 157-194. https://doi.org/10.1111/j.0887-378X.2004.00305.x 
Suwanwela, N.C. 2014. Stroke epidemiology in Thailand. Journal of Stroke. 16(1): 1-7. https://doi.org/10.5853/jos.2014.16.1.1

Suwapan, D., Taechaarpoenkul, W., Archong, Y., Paramakom, A., Theppanom, C., Kongkerd, P., Ruamsuk, S., Posawang, P., Saypan, Y. 2005. Sirindhron National Medical Rehabilitation Center-Functional Assessment (SNMRC - Functional Assessment). Bulletin of the Department of Medical Services. 30(1): 23-28.

Turner, R.C., and Carlson, L. 2003. Indexes of item-objective congruence for multidimensional items. International Journal of Testing. 3(2): 163-171. https://doi.org/10.1207/S15327574IJT0302_5

Walter-Ginzburg, A., Guralnik, J.M., Blumstein, T., Gindin, J., and Modan, B. 2001. Assistance with personal care activities among the old-old in Israel: a national epidemiological study. Journal of the American Geriatrics Society. 49(9): 1176-1184. https://doi.org/10.1046/j.1532-5415.2001. 49234.x

Wolf, T.J., and Rognstad, M.C. 2013. Changes in cognition following mild stroke. Neuropsychological Rehabilitation. 23(2): 256-266. https://doi.org/ 10.1080/09602011.2012.748672

World Federation of Occupational Therapists. Definitions of occupational therapy from member organizations. 2013. 